\title{
Long-term photometric study of LBVs in M33
}

\author{
A. Zharova ${ }^{1}$, O. Sholukhova ${ }^{2}$ \\ ${ }^{1}$ Sternberg Astronomical Institute, Moscow, Russia \\ 2 Special Astrophysical Observatory of Russian Academy of Sciences
}

The extensive plate collection of Sternberg Astronomical Institute (Moscow) was used for a study of the photometric behaviour of bright variable stars in M33. We present here LBV V268 ( $\operatorname{VarC}$ ) and the LBV-candidate $\mathrm{H} \alpha 7$. About 600 magnitude estimates were obtained for each star for 1973 - 2002. The light curves of both stars are typical for Luminous Blue Variables. V268 shows large and complex quasiperiodic variations from 2 to 3400 days. In $\mathrm{H} \alpha 7$, we found several quasi-periods from 20 to 160 days. Amplitudes of these variations depend on the state of activity of the stars.

The historical light curve of V268 combined with the new data is shown in Fig.1. We see the photometric behaviour typical for LBVs. 1) The star's magnitude varies with an amplitude of about 0.2-0.3 mag on time scales from weeks to months. 2) There were few bright states (outbursts) with amplitudes of 1-2 mag on time scales of decades. There are low and high states in the light curve and the amplitude of the variations depends on the state. In total, we found 12 periods ranging from 2 days up to 3400 days.

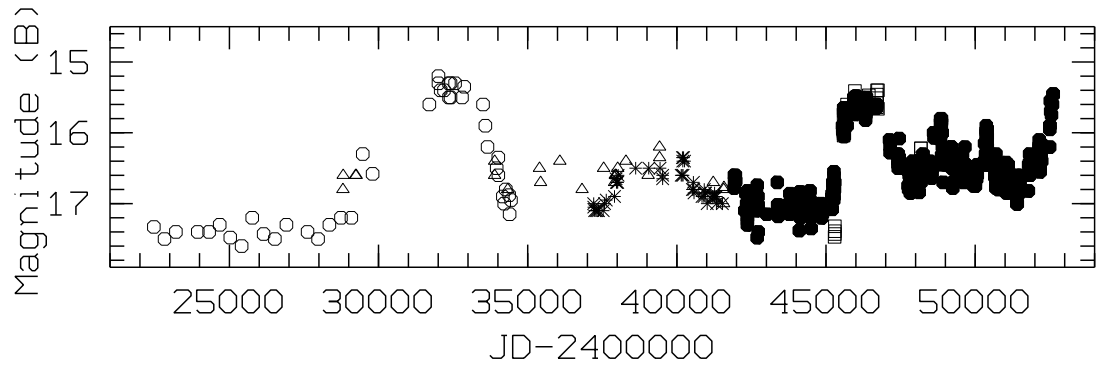

Figure 1: Light curve of V268. Open circles are the data of Hubble and Sandage (1953); triangles, the data of Sharov (1973); asterisks, the data of Rosino and Bianchini (1973); squares, the data of Kurtev et al. (1999); filled circles, our estimates.

Acknowledgments. This study was supported by a grant from the Programme of Support for Leading Scientific Schools of Russia No. 389-2003-2, and RFBR grant No. 0302-16341. O. Sholukhova has been supported by INTAS grant YSF 2002-281. The authors are grateful to S.Fabrika and N.Samus for useful remarks.

\section{References}

Hubble E., Sandage A., 1953, ApJ, 118, 353

Kurtev R.G., Corral L.J., Georgiev L., 1999, A\&A, 349, 796

Rosino L., Bianchini A., 1973, A\&A, 22, 453

Sharov A.S., 1973, Perem. Zvezdi, 19, 3 